\title{
Direct Calculation of the Free Energy Using Reduced Boltzmann Factors: Application to Lennard-Jones Fluids and Square-Well Fluids
}

\author{
Seung Do Hong* and Du-Jeon ,Jang \\ School of Chemistr; Seoul National University, Seont 151-742. Korea \\ Received Aprit 28, 2003
}

Key Words : Free energy, Entropy, Reduced Boltzmann factor, Direct calculation

Reduced Boltzmann factors have been introduced and averaged for the direct calculation of free energy during a single molecular dynamics or Monte Carlo simulation. Very stable results have been obtained for the excess free energies of the Lennard-Jones fluids and the square-well fluids at high densities.

The free energies and entropies of chemical and biological systems are very important thermodynamic properties related to the direction of spontaneous change. A number of methods for the calculation of the free energy and entropy has been proposed with rapid progress in the field of computer simulations. ${ }^{1-3}$ It is very important to compute the free energy and entropy at high densities because a number of chemical and biological processes occur in the highdensity region. However, it has been difficult to make these calculations directly from computer simulations. Recently a fluctuating cell model ${ }^{4}$ using the Metropolis algorithm for the generation of the constant NVT ensemble was proposed, although its application is limited to high-density phases of two-dimensional hard dumbbells. Although it is actually impossible to calculate the free energies directly from the averages of the Boltzmann factors owing to large fluctuations, ' the fluctuation problem can be eased considerably by the multicanonical ensemble method ${ }^{6}$ or the multiensemble sampling method. ${ }^{\text {? }}$

On the other hand, a practical method using the canonical ensemble average of effective acceptance ratios (the EAR method) was proposed for the direct calculation of the free energy. In the EAR method, large fluctuations according to the evaluation of the excess entropy can be reduced considerably by using the effective acceptance ratio, which is expressed as

$$
f\left(r_{k}, r^{*}\right)- \begin{cases}\exp \left[-\left(\phi_{k}-\phi\right) / k T\right] & \text { if } \phi_{k} \geq \phi, \\ 1+2.3\left\{\exp \left[-\left(\phi_{k}-\phi\right) / 2 k T\right]-1\right\} & \text { if } \phi_{k}<\phi,\end{cases}
$$

where $\phi$ is the potential energy of a molecule at $r$, a

"Corresponding author. E-mail: sdhong@ssnuac.kr; Fax: -82-2889-1568 configuration sampled during the constant $\mathrm{NVI}$ molecular dynamics $^{2}$ or the Metropolis Monte Carlo simulation, and $\phi_{R}$ is the potential energy of the molecule at $r_{k,}$ a virtual random configuration generated by a separate parallel Monte Carlo procedure within the cell $\Delta$. The cell was selected as a cube of a fixed volume centered at $r$.

To reduce the fluctuations further, we could use scaled acceptance ratios ${ }^{9}$ and acceptance ratios. ${ }^{10}$ Because scaled acceptance ratios are obtained from acceptance ratios, both of these methods ${ }^{9.10}$ exploit the averages of acceptance ratios. The methods, while stable, inevitably have limitations owing to the value of the acceptance ratio confined to 1 . In the present work, we try to use an effective function, " that is intermediate between the acceptance ratio and the Boltzmann factor. We rename the effective function as the reduced Boltzınann factor, which is given by

$$
B_{r w d}\left(r_{k, r}\right)- \begin{cases}\exp \left[-\left(\phi_{k}-\phi\right) / k T\right] & \text { if } \phi_{k} \geq \phi \\ \exp \left[-\left(\phi_{k}-\phi\right) / 2 k T\right] & \text { if } \phi_{k}<\phi\end{cases}
$$

The reduced Boltzmann factor is obtained by replacing a factor of 2.3 in the effective acceptance ratio ${ }^{8}$ with a factor of

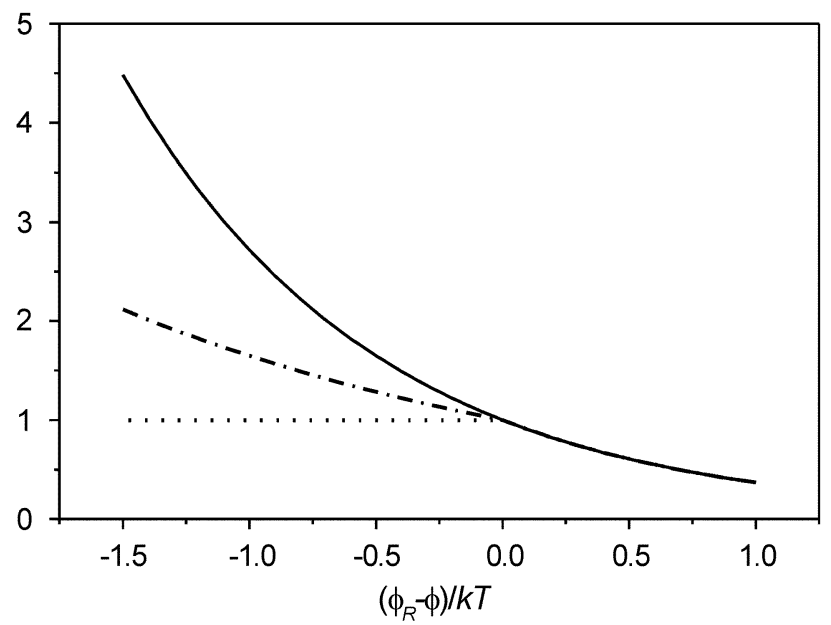

Figure 1. The reduced Boltzmann factor (dash-dotted), the Boltzmann factor (solid). and the acceptance ratio (dotted) with the variation of the potential energy diflerence $\left(\phi_{k}-\phi\right)$. 


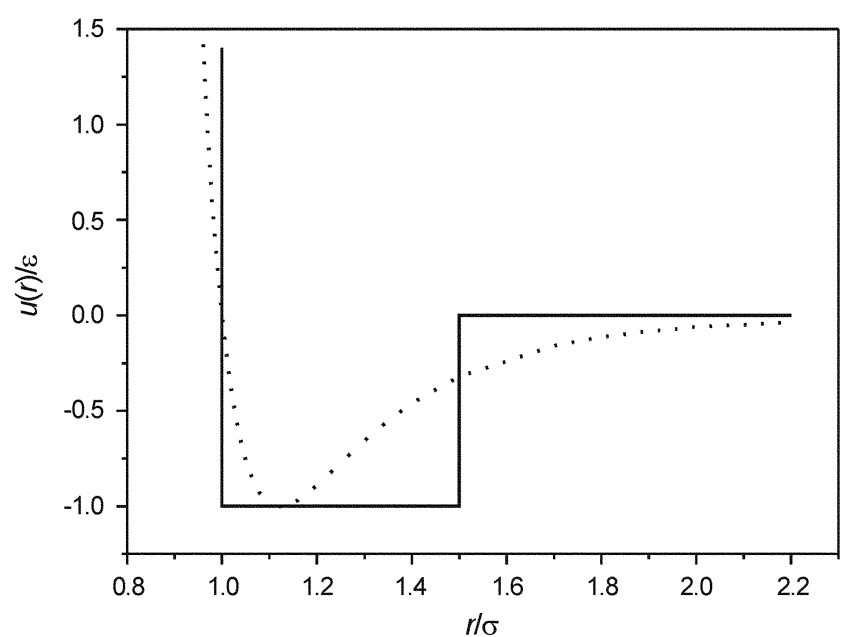

Figure 2. 'The I, enmard-Jones polential (dotted) and the square-well potential (solid).

1. The reduced Boltzmann factor is shown with the Boltzmann factor and the acceptance ratio in Figure 1.

In this work, the excess entropy of an $N$-particle system is approximately expressed as

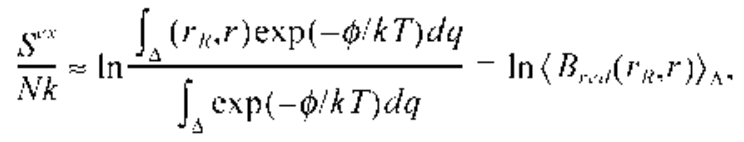

where $\left\langle B_{r \text { cis }}\left(r_{k}, r\right)\right\rangle_{\Delta}$ denotes the canonical ensemble average of $B_{r e d}\left(r_{k, r}\right)$ over $r_{k *}$. This method employing the average of $B_{r, d}\left(r_{N}, r\right)$ will be named a reduced Boltzmann factor (RBF) method. We have applied here the RBF method to the constant $N V I$ molecular dynamics and the Metropolis Monte Carlo simulations for the high-density phases of the Lennard-Jones fluids ${ }^{12.13}$ and the square-well fluids. ${ }^{14}$ The Lennard-Jones potential and the square-well potential are shown in rigure 2. The upper and lower limits of the cell volumes suggested in the acceptance ratio averaging (ARA) method were $0.56 \mathrm{~V} / \mathrm{N}$ and $0.27 \mathrm{~V} / \mathrm{N}$, respectively. ${ }^{10}$ Considering that the $\mathrm{RBF}$ method overestimates the excess entropy compared with the ARA method, we chose the cell volumes for the Lennard-Jones fluids and the square-well fluids as $0.69 \mathrm{~V} / \mathrm{N}$ and $0.55 \mathrm{~V} / \mathrm{N}$, respectively.

In each configuration sampled during the Metropolis Monte Carlo simulation, a sampled molecule was moved to a virtual random configuration, ${ }^{\prime} \%$, through a separate parallel Monte Carlo procedure. Then $B_{r e d}\left(r_{h}, r\right)$ was estimated at $r_{k}$ and averaged over the canonical ensemble. Thereafter, the virtual configuration was removed to perform the normal Metropolis Monte Carlo procedure. In the case of the constant NVI molecular dynamics simulation, $B_{r e d}\left(r_{k} r\right)$ was calculated and averaged at each time step through the separate parallel Monte Carlo procedure as well. The excess Helınholtz free energy was obtained by

$$
A^{2 y}-U \text { IS }
$$

where $U$ is the averaged configurational energy.

A total of 216 molecules were used for simulations of the
Table 1. Excess Helmholtz frec encrgies $\left(-f^{2 \tau} / \lambda k T\right)$ of the 1.ennard-Iones lluids on the isothems $T^{*} \quad 0.75,1.15$, and 1.35

\begin{tabular}{ccccccc}
\hline$T^{*}$ & $\rho^{*}$ & $\mathrm{RBF}^{\prime}$ & $\mathrm{TI}^{6}$ & $\mathrm{EAR}^{c}$ & $\mathrm{WC}^{d}$ & $\mathrm{MPC}^{\mathrm{c}}$ \\
\hline 0.75 & 0.6 & 3.69 & 3.73 & 3.72 & 3.65 & - \\
& 0.7 & 4.17 & 4.17 & 4.16 & 4.14 & - \\
& 0.8 & 4.48 & 4.47 & 4.49 & 4.46 & - \\
& 0.84 & 4.56 & 4.53 & 4.54 & 4.51 & 4.59 \\
1.15 & 0.6 & 1.68 & 1.78 & 1.73 & 1.74 & - \\
& 0.65 & 1.78 & 1.84 & 1.81 & 1.82 & - \\
& 0.75 & 1.85 & 1.89 & 1.86 & 1.88 & 1.91 \\
& 0.85 & 1.82 & 1.78 & 1.78 & 1.77 & 1.79 \\
& 0.6 & 1.16 & 1.26 & 1.21 & 1.22 & - \\
& 0.7 & 1.22 & 1.29 & 1.26 & 1.26 & - \\
& 0.8 & 1.17 & 1.19 & 1.16 & 1.16 & - \\
\hline
\end{tabular}

"Results calculated usine the reduced Boltzmann factor" method. "Results of thermodynamic integration trom refs. 12 and 13. "Results of the effective acceptance ratio method from ref. 8. "Results of the perturbation theory from rel. 15. "Results of the multi-particle cortelation from ref." 16.

Table 2. Lixcess [lumholtz fres encrgies $\left(-f^{* x} / N k J\right)$ of the

\begin{tabular}{|c|c|c|c|c|c|}
\hline$\rho^{*}$ & $T *$ & $\mathrm{RI}|3|^{* \prime}$ & $\mathrm{TT}^{\mathrm{h}}$ & $\mathrm{I}: \wedge \mathrm{R}^{\circ}$ & WCAd \\
\hline \multirow[t]{3}{*}{0.88} & 1.095 & 1.98 & 1.99 & 2.01 & 1.94 \\
\hline & 0.94 & 2.92 & 2.88 & 2.92 & 2.84 \\
\hline & 0.591 & 6.97 & 6.86 & 6.90 & 6.80 \\
\hline \multirow[t]{5}{*}{0.85} & 2.202 & -0.37 & -0.37 & -0.41 & -0.44 \\
\hline & 1.128 & 1.92 & 1.91 & 1.89 & 1.86 \\
\hline & 0.880 & 3.36 & 3.35 & - & 3.32 \\
\hline & 0.760 & 4.45 & 4.44 & - & 4.41 \\
\hline & 0.591 & 6.84 & 6.81 & 6.87 & 6.74 \\
\hline \multirow[t]{3}{*}{0.75} & 2.849 & -0.58 & -0.50 & - & -0.56 \\
\hline & 1.071 & 2.19 & 2.25 & - & 2.21 \\
\hline & 0.881 & 3.28 & 3.30 & - & 3.27 \\
\hline
\end{tabular}
Lennard-lones fluids on the isochores $\rho^{*}=0.88 .0 .85$. and 0.75

"Results calculated using the reduced Boltzmann faketor method. "Results of themodynamic integration from ref. 13. "Results of the eflective acceptance ratio method from rel. 8. "Results of the perturbation theory from ref. 15 .

Lennard-Jones fluids and the square-well fluids. About $2 \times 10^{6}$ configurations were averaged after equilibration using the periodic boundary condition, the long-range energy correction, and the cut-off distance of half the box length. For the liquid-gas coexistence region, only Monte Carlo simulations were carried out and we used the method of Hansen and Verlet. ${ }^{12}$ which forced the system to remain homogeneous.

The excess Helmholtz free energies calculated using the $\mathrm{RBF}$ method along the isotherms of $T^{*}=0.75,1.15$, and 1.35 for the Lennard-Jones fluids are listed in Table 1 . The results are compared with those from thermodynamic integration $^{12.15}$ (II), the EAR method, ${ }^{*}$ the perturbation theory by Weeks, Chandler, and Anderson ${ }^{15}$ (WCA), and the multiparticle correlation ${ }^{16}$ (MPC). The free energies calculated along the isochores of $\rho^{*}=0.88,0.85$, and 0.75 for the Lennard-Jones fluids are listed in Table 2. The results are compared with those from $T 1,{ }^{13}$ the EAR method, and the 
Table 3. Exicess Helmholtz free energies $\left(-A^{e x / R} / k T\right)$ of the squarewell fluids on the isochore $p^{*}=0.68$

\begin{tabular}{cccc}
\hline$T^{*}$ & RBF $^{\text {r }}$ & TDSMC $^{5}$ & EAR $^{\circ}$ \\
\hline 1.053 & 2.26 & 2.25 & 2.04 \\
1.081 & 2.12 & 2.12 & 1.89 \\
1.111 & 1.99 & 1.99 & 1.76 \\
1.143 & 1.86 & 1.86 & 1.62 \\
1.176 & 1.73 & 1.73 & 1.48 \\
1.212 & 1.59 & 1.60 & 1.36 \\
1.250 & 1.45 & 1.46 & 1.20 \\
1.290 & 1.32 & 1.34 & 1.06 \\
\hline
\end{tabular}

"Results calculated using the reduced Boltzmann factor method. "Results of the temperature-and-density-scaling Monte Carlo method from ref. 14. 'Results calculated using the effective acceptance ratio method from ref. 8 .

WCA perturbation theory. ${ }^{15}$ The results calculated along the isochore $\rho^{*}=0.68$ for the square-well fluids are also compared with the results of the temperature-and-densityscaling Monte Carlo (TDSMC) method ${ }^{1+}$ and the results calculated using the EAR method in Table 3.

The RBF method bridges a gap between the ARS method ${ }^{8}$ and the ARA method. ${ }^{10}$ In the EAR method. significant fluctuations remain. In the ARA method, biased acceptance ratios are averaged for $\phi_{R}<\phi$. The $\mathrm{RBF}$ method reduces the residual fluctuation in the EAR method as well as the bias in the ARA method.

Whereas the EAR method does not work well for the square-well fluids, the results calculated using the RBF method agree well with reported results for the high-density phases of the Lennard-Jones fluids and the square-well fluids. Here. it is noteworthy that the Lennard-Jones potential is obviously different from the square-well potential. Thus it is very important that the RBF method works well for both the Lennard-Jones fluids and the squarewell fluids. The averages of $B_{\text {red }}\left(r_{R}, r\right)$ were more stable compared with those of effective acceptance ratios. ${ }^{s}$ The relative error of the RBF method in the calculation of the excess entropy is estimated to be within $1 \%$, whereas that of the EAR method was as large as $2 \%$. Even though $B_{r e d}\left(r_{R}, r\right)$ has been averaged over the canonical ensemble. this approach has the character of the grand canonical ensemble because a sampled molecule is subtracted and inserted through the virtual random sampling.

On the other hand. real fluids would be well modeled with a three-dimensional soft intermolecular potential compared with a two-dimensional hard one. Whereas the application of the fluctuating cell model ${ }^{4}$ has been restricted only to twodimensional hard dumbbells. the RBF method has been successfully applicable to more realistic three-dimensional Lennard-Jones fluids and square-ivell fluids. The absolute excess free energy is calculated instead of the relative one because this approach does not need any reference system except the ideal gas. Our method overcomes the difficulties arising from the phase transitions of ligh-density fluids or solids as well. ${ }^{17}$ The RBF method is very efficient because the excess free energy is directly calculated from a single computer sinulation at fixed density and temperature.

We have calculated directly the excess free energies of the Lennard-Jones fluids and the square-well fluids from the averages of reduced Boltzmann factors during a single conputer simulation. Very stable results have been obtained for both the Lennard-Jones fluids and the square-well fluids at high densities. Studies on complex systems are currently undenvay using this method.

Acknowledgment. The Brain Korea 21 Program and the Center for Molecular Catalysis are acknowledged for their financial support.

\section{References}

1. Valleau. .T. P.: Torrie. G. M. In Modern Theoretical Chentistr: Berne. B. T.. Ed.: Plenum Press: New York. U. S. A.. 1977: Vol. 5. p 169.

2. Allen. M. P.: Tildesley. D. J. Computer Simulation of Liquids' Clarendon: Oxford. U. K.. 1987; p 213.

3. Panagiotopoulos. A. Z. Fluid Phase Equilit. 1996, 116.257.

4. Gay. S. C.: Rainwater. J. C.: Beale. P. D. J. Chent. Phis. 2000. 112.9841.

5. Metropolis. N.: Rosenbluth. A. W.: Rosenbluth. M. N.: Teller. A. H: Teller. E. J. Chem. Phys. 1953, 21. 1087.

6. (a) Berg. B. A.; Neuhaus, T. Phys. Rev Lett 1992, 68, 9. (b) Lee. J. Phys. Ran Lett. 1993. 71, 211. (c) Jang. S.; Pak. Y.: Shin. S. J. Chent. Phis $2002,116.4782$.

7. Han1. K.-K.: Kiml. K. H.: Mhin. B. T.: Son1. H. S. J. Comput Chent 2001. 22. 1004 .

8. (a) Hong. S. D.: Thon1. M. S. Chem. Phus. Lett. 1997. 273 . 79. (b) Hong. S. D.; Yoon, B. J.: Jhon, M. S. Chem. Phus, Lett 1992, 188. 299

9. Hong. S. D.: Jang. D.-J. Chent. Lett. $2002,442$.

10. Hong. S. D.: Tang. D.-T. Chen. Lett 2002. 946.

11. Hong. S. D. Bull Korean Chem. Soc. 2000. 21.697.

12. Hansent. T.P.: Verlet. L. Plys. Rev 1969.184. 151

13. Levesque. D.: Verlet. L. Phys. Rev 1969. 182, 307.

14. Brilliantov. N. V: Valleau. J. P. J. Chem. Phys. 1998, 108. 1115

15. Weeks, J. D.; Chandler. D; Anderson, H. C.J. Chem. Phys 1971. 5+. 5237.

16. Giaquitita. P. V: Giunta. G.: Prestipino Giarritta. S. Phos Ret .A 1992. 45.6966 .

17. Yoon, B. J: Hong, S. D; Jhon, M. S.; Scheraga. H. A. Chem. Phus. Lett 1991. 181.73. 